\title{
STENT-GRAFT IMPLANTATION THROUGH PARTIAL STERNOTOMY
}

Mikio Ninomiya, MD, Shinichi Takamoto, MD, Yutaka Kotsuka, MD, Takeshi Miyairi, MD, and Munemoto Endo, MD,

\section{Tokyo, Japan}

In the field of thoracic aortic surgery, minimally invasive approaches have not commonly been used. We report a case of distal arch aortic aneurysm treated with a new approach for stent-graft implantation through partial sternotomy.

Clinical summary. A 29-year-old woman who had been involved in a traffic accident in which she was hit in the chest 2 years previously was given a diagnosis of distal arch aortic aneurysm and was referred to our hospital. The patient had a history of frequent attacks of severe asthma and had been hospitalized for steroid therapy twice within the previous year, despite regular use of bronchodilators. Angiography revealed a 50-mm saccular aneurysm in the distal arch. Although catheter insertion of a stent graft is the choice for a high-risk patient in our department, it was not indicated for this patient because the aortic lumen just proximal and just distal to the aneurysm was narrow and the aorta just proximal to the aneurysm curved sharply, making the possibility of a postoperative endoleak high (Fig 1). The patient, a young woman, had no symptoms relating to the aneurysm and very strongly insisted that the length of the skin incision be minimal. We decided to perform stent-graft implantation by using Kato's procedure ${ }^{1}$ through partial sternotomy rather than the conventional graft replacement through a left thoracotomy to avoid direct mechanical damage to the lung caused by thoracotomy and to meet the patient's cosmetic request.

An 11-cm midline skin incision was made, followed by an Lshaped sternal incision from the sternal notch into the left fourth intercostal space. The ascending aorta, the proximal half

From the Department of Cardiothoracic Surgery, Faculty of Medicine, University of Tokyo, Tokyo, Japan.

J Thorac Cardiovasc Surg 2001;121:992-3

Copyright (C) 2001 by The American Association for Thoracic Surgery

$0022-5223 / 2001 \$ 35.00+0 \quad \mathbf{1 2 / 5 4 / 1 1 0 4 8 8}$

doi: $10.1067 / \mathrm{mtc} .2001 .110488$

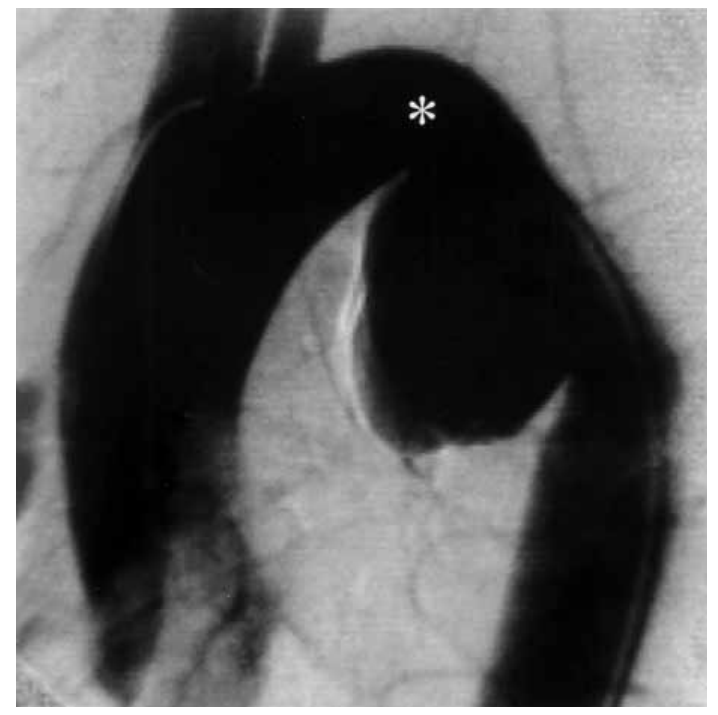

Fig 1. Preoperative digital subtraction angiography revealed a saccular aneurysm in the distal arch. The aortic lumen both just proximal and just distal to the aneurysm was narrow, and the aorta between the left subclavian artery and the aneurysm (*) curved sharply.

of the aortic arch, and the right atrial appendage were exposed. Cardiopulmonary bypass was instituted between the ascending aorta and the right atrium by means of a single venous cannula. Because the distal arch, which had been planned to be incised, was not sufficiently visible, a semicircumferential incision was made into the proximal aorta around the root of the brachiocephalic artery under circulatory arrest and retrograde cerebral perfusion (Fig 2). Retrograde cerebral perfusion was conducted by increasing the central venous pressure up to around $18 \mathrm{~mm}$ 


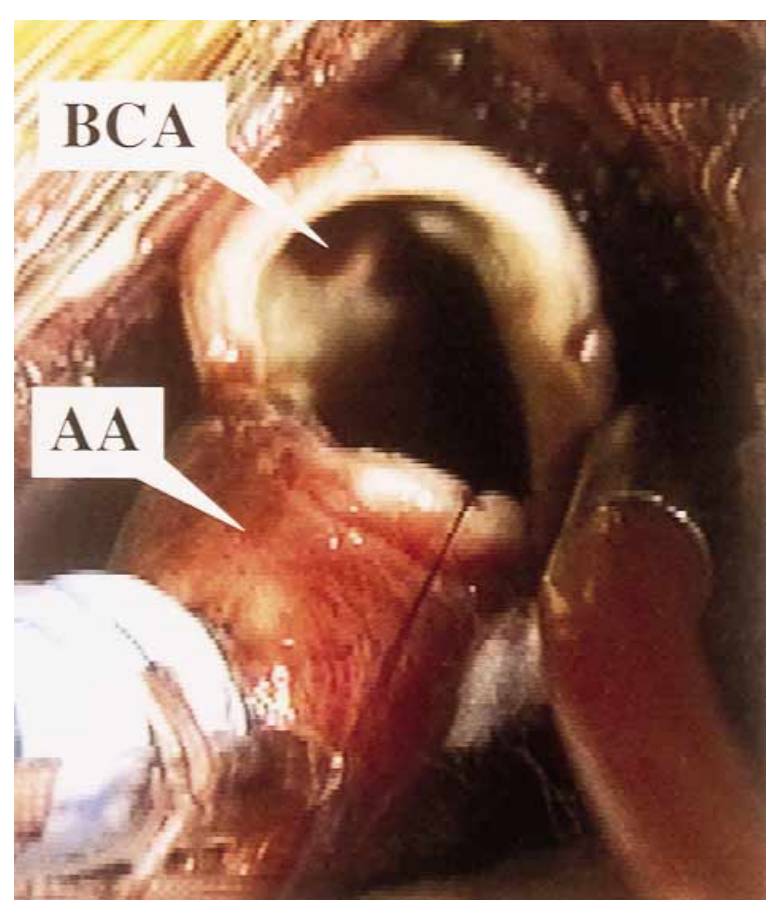

Fig 2. A semicircumferential aortotomy was made around the root of the brachiocephalic artery. Through this incision, the lumen of the distal arch was clearly visible. $B C A$, Brachiocephalic artery; $A A$, ascending aorta.

$\mathrm{Hg}$, as we usually do when selective cannulation into the superior vena cava is difficult, and sufficient retrograde flow from the cervical vessels was obtained. From the aortotomy, the orifice of the aneurysm and the descending aorta was clearly visible. A stent graft made of a 30-mm woven Dacron graft and a $30 \times 50-\mathrm{mm} \mathrm{Z}$ stent was easily inserted, and its proximal end was anastomosed circumferentially to the aorta just distal to the left subclavian artery by the inclusion technique.

Although intraoperative ultrasonography revealed no abnormal findings, a pressure gradient between the upper and lower extremities appeared postoperatively. Angiography conducted on the third postoperative day revealed an endoleak from the greater curvature side of the proximal anastomosis, making the graft stenotic. A bare $30 \times 75-\mathrm{mm} \mathrm{Z}$ stent was inserted intraluminally, and the endoleak and pressure gradient completely disappeared. No respiratory complications occurred. The patient was satisfied with the result and is well a year after the operation.

Discussion. Although minimally invasive cardiac surgery has been aggressively performed, it has not been popular in the field of aortic surgery. The use of stent grafts is thought to make minimally invasive approaches practicable for aortic operations. In our case we used an L-shaped sternal incision, which was used by Svensson ${ }^{2}$ for coronary artery bypass grafting, and our original semicircumferential incision into the proximal aorta mainly to meet the patient's cosmetic requirement. As far as less invasiveness is concerned, we realize that it may be controversial whether our method, which required circulatory arrest, was truly less invasive for the patient's pulmonary function than the con- ventional graft replacement through a left thoracotomy. Our method, however, was very effective and supplied good visualization of the lumen of the distal arch, making it possible to complete the operation without full sternotomy or thoracotomy, and it did not cause any postoperative respiratory problems. This method can be applied to most patients requiring surgical implantation of a stent graft into the distal arch (Kato's procedure) and even to patients requiring Kato's procedure and concomitant procedures, such as coronary artery bypass grafting, an aortic valve operation, or an ascending aortic operation. The main cause of the postoperative endoleak in this patient was our inexperience in this method, and we believe complications like this can be prevented with more experience and more careful operative maneuvers.

Received for publication July 20, 2000; accepted for publication July 28, 2000.

Address for reprints: Mikio Ninomiya, MD, 7-3-1 Hongo, Bunkyo-ku, Tokyo 113-8655, Japan (E-mail: mikio.ninomiya@nifty.ne.jp).

\section{REFERENCES}

1. Kato M, Ohnishi K, Kaneko M, Ueda T, Kishi D, Mizushima T, et al. New graft-implanting method for thoracic aortic aneurysm or dissection with a stented graft. Circulation 1996;94(Suppl):II188-93.

2. Svensson LG. Minimal-access “J” or "j" sternotomy for valvular, aortic, and coronary operations or reoperations. Ann Thorac Surg 1997;64:1501-3. 